\title{
Research on the Increase of Mineral Dissociation Degree by High Pressure Water Jet
}

\author{
Mingming Cai ${ }^{1}{ }^{*}$, Chao Xu${ }^{1}$, Tengyue $\mathrm{Gao}^{1}$, Juntong Zhang ${ }^{1}$, Luzhong Bo ${ }^{1}$, Yanbo Chen ${ }^{1}$, Guanglin Qin ${ }^{1}$, Qiang $\mathrm{Ji}^{1}$, \\ Congquan $\mathrm{Yu}^{1}$ \\ ${ }^{1}$ Metallurgical Laboratory Branch of Shandong Gold Mining Technology Co., Ltd., Laizhou, 261400, China
}

\begin{abstract}
The traditional grinding technology of ball mill has many problems, such as high energy consumption, unable to maintain the original crystal shape, and it is difficult to obtain high quality and high purity ultrafine powder. The high-pressure water jet mineral dissociation technology is a new technology which is developed in response to these requirements. The experiment shows that the water jet technology has a strong ability of ultrafine grinding. With the increase of jet velocity, the crushing effect is more obvious. The high-pressure water jet pulverization is superior to ball milling for the separation of rare earth minerals, which has the characteristics of high dissociation degree and relatively uniform particle size. The water jet pulverization is more beneficial to the separation and recovery of rare earth minerals.
\end{abstract}

\section{Introduction}

In recent years, superfine grinding technology of solid materials has become one of the most important basic technologies to support high-tech industry. Solid materials that need to be broken cover a wide range of fields, including non-metallic minerals, solid waste, pigments, food, medicine, catalysts, magnetic memory elements, coal water slurry, advanced abrasives and so on. After crushing, the economic value of some kinds of solid materials will be greatly improved, and some kinds will be more convenient for processing and reuse. Therefore, the crushing of solid materials has become an essential process to improve its economic value, processing technology, utilization efficiency and resource utilization.

The superfine powder of solid material is used in almost all sectors of the national economy, involving all fields. In the field of environmental protection, solid material crushing is used in the recycling of solid waste and various powdery sewage treatment agents. In the field of papermaking, solid material grinding is used in the preparation of pulp, coating superfine pulp for papermaking, etc. In the field of mining, solid material crushing is used in grinding of metal ore, crushing and deep processing of non-metallic minerals, comprehensive utilization of low-grade minerals, extraction of useful elements in tailings, etc. In the field of metallurgy, solid material crushing is used in powder metallurgy, mechanical alloying, metallurgical raw material treatment, metallurgical waste utilization, etc. In the field of machinery, solid material grinding is used in particle size sand, casting sand, materials, solid lubricants and so on. In the field of agriculture, solid material crushing is used in grain processing, chemical fertilizer production, powder pesticide, soil improvement, various additives in beverages, artificial rainfall accelerator and so on. In the field of rubber, solid material crushing is applied to solid fillers, reinforcing materials, functional fillers, etc. In the field of plastics, solid material crushing is used in the preparation of plastic raw materials, powder plastics, etc. In the field of printing, solid material grinding is used in ink production, copper gold powder, ink-jet printing cartridge, etc. In the field of chemical industry, solid material crushing is used in the treatment of raw materials and catalysts.

\section{Mineral dissociation technology}

\subsection{Mineral dissociation technology by ball mill}

At present, as the main crushing equipment, ball mill is widely used in the processing and metallurgy of metal and non-metal ores, as well as the crushing part of some basic industries in the field of chemical building materials. Ball mill can be widely used since it was invented, mainly because of its stable work, strong adaptability to materials, and little requirement for working environment. It can adapt to the operation under different conditions, and can meet the needs of most industrial crushing, and it is easy to adjust the product fineness of abrasive materials. From the principle and a large number of experiments, the movement of the ball in the ball mill cylinder is mainly determined by the rotation speed of the ball mill cylinder, the diameter ratio of the ball and the filling rate. The ball mill can be operated by both dry and wet methods. It has the advantages of simple structure, reliable operation, long-term operation, good sealing, negative pressure operation and dust prevention. At the same time, the ball

*Corresponding Author: Mingming Cai

zxbdhh@vip.qq.com 
mill also has a fatal disadvantage, low efficiency. The ball mill has strong adaptability to materials and large production capacity, which can meet the requirements of modern industrial production. The crushing ratio is large, which can reach more than one goal, and it is easy to adjust the fineness of grinding products. It can adapt to the operation in different situations, either dry or wet operation, or the combination of drying and wet operation at the same time. Simple structure, solid, reliable operation, convenient maintenance and management, long-term operation, good sealing, negative pressure operation, prevent dust flying. However, the ball mill also has some major shortcomings, such as low work efficiency. The power consumption of hard material grinding accounts for about $70 \%$ of the total power consumption of the plant, while the effective utilization rate of power used for grinding is only about $5 \%$, and most of the rest of the power is converted into heat consumption. In addition, the ball mill is bulky, and the total weight of large mill can reach several hundred tons.

\subsection{Mineral dissociation technology by high pressure water jet}

Traditional comminution technology has high energy consumption and low comminution efficiency. Therefore, how to save a lot of energy from the crushing process has been the goal of people's research. In addition, new material technology has put forward higher and higher requirements for ultra-fine powder. For example, ultrafine particles should not only have narrow size and high purity, but also maintain the original crystalline shape and surface lustre of particles. The high-pressure water jet comminution technology is a new technology for the preparation of ultrafine powder. High pressure water jet comminution simply means that the highly concentrated water jet acts on the comminated material in a certain way, and produces pressure transients in the cracks and cleavage surface of the material to comminute the material. Because high pressure water jet usually has a very high speed and highly concentrated energy, the loading time is short and the energy density of the load is high. In addition, cavitation can be formed in the crushing process, so the main crushing mode of the material is cleavage crushing. Due to the good cleavage of water jet, high-pressure water jet can not only reduce the energy consumption, but also prepare high-quality ultra-fine powder. High pressure water jet comminution of mica is a kind of practical superfine comminution technology. It can not only meet the fineness requirements of Pearlescent Mica Powder, but also protect the flake structure and crystal surface gloss of mica. The superfine mica powder is mainly used as ingredient or additive for paint, rubber, glass fibre reinforced plastics, ceramics, plastics, coatings, etc. it can also be used to produce mica paper and crystal seed for producing synthetic mica. Adding water as the medium in the process of high-pressure water jet wet grinding can better protect the crystal surface lustre of mica flakes. Compared with dry grinding, it has more extensive utilization and higher economic value. High pressure water jet ultra-fine grinding technology is a new and most promising technology in ultra-fine grinding technology. It is of great practical significance to study the high-speed water jet ultra-fine grinding technology as soon as possible and create a new way to prepare ultra-fine powder with high efficiency and low price for the development and resource utilization of ultra-fine grinding industry in China.

\section{Mineral dissociation device by high pressure water jet}

This experiment uses a self-developed high-pressure water jet mineral crushing device, which is mainly composed of power device, crushing device and separation device.

\subsection{Power device}

High pressure piston pump is used as power unit. The main function of the plunger pump is to pressurize the feed water, so that it has a greater pressure. There are two check valves on a plunger pump, and the direction is opposite. When the plunger moves in one direction, there is negative pressure in the cylinder. At this time, one check valve opens and the liquid is sucked into the cylinder. When the plunger moves in another direction, the liquid is compressed and the other check valve opens, and the liquid sucked into the cylinder is discharged. After continuous movement of this working mode, continuous oil supply, water and air are formed. It can work under high pressure continuously. The design of over balanced pressure compensation enhances the stiffness of hydrostatic bearing of friction pair and overcomes the problem of abrasion and scuffing of friction pair. It not only greatly enhances the shock resistance and pollution resistance of the pump, but also reduces the wear. So that the life of the pump is prolonged. The core material of the pump is ductile iron with special heat treatment process, which greatly improves the heat resistance and wear resistance of the main moving parts. At the same time, the plunger adopts ceramic glaze, which further ensures the service life of the pump.

\subsection{Crushing device}

Due to the long working time in industrial application, the unidirectional target is easy to be damaged and replaced frequently. The advantage of this method is not only to solve the problem of target material loss and frequent replacement, but also to increase the processing capacity. In the process of operation, there will be no spark, and no harmful gas or suspended solids will be released due to thermal reaction. Not in operation, little dust, no chemical pollution, can reduce noise to the greatest extent, provide a clean and quiet working environment for workers. It can crush materials that cannot be crushed or should not be crushed by ordinary mechanical crushing equipment, such as various metal oxides, explosives, flammable and explosive products, etc. Due to the water wedge effect, when the material is crushed into small particles, the different components in the material are separated from 
each other, so the product with high purity can be obtained. Due to the short grinding time and no medium in the grinding process, no new impurities and no pollution will be introduced.

\subsection{Separation device}

The main function of the separation device is not only to separate the material particles in the experiment from the water flow, but also to screen out different grades according to the needs, and at the same time to play a certain concentration role. For the particles that do not meet the requirements of slurry, they can be returned to the system for secondary grinding. The selection and matching of the separation system need to meet the following requirements to meet the needs of the whole system flow. In the design of the separation system, priority should be given to the flow demand. In the production, all the flow should pass through the separation system, so as to ensure that all the materials can be screened out in the experiment and avoid the waste of materials. Meet the needs of screening particle size. The screened material is easy to clean. That is, the screening equipment is required to be able to conveniently collect the crushed materials. Because this experiment is only a basic experiment to modify the model in the future, we need to control the cost. At present, more mature hydraulic separation devices include cyclone separator, sand funnel and traditional vibrating screen. After comprehensively considering the advantages and disadvantages of these kinds of separation equipment, the self-designed multistage hydraulic screen is selected. The multi-stage screening separation device used in this experiment is to let the mixed liquid pass through different standard sieves, and the water flow with a certain flow rate directly pours on the screen net. At the same time, it flows down each screen net to separate it into several particle sizes, and then weigh them respectively to obtain the mass percentage to express the distribution of particles.

\section{Experimental research of mineral dissociation degree}

\subsection{Selection of experimental mineral}

High pressure water jet is mainly used in the superfine flaking of kaolin, mica and other materials, and put into the industrial application of preparing Pearlescent Mica Powder. The production of mica powder can be divided into dry process and wet process. It is easy to destroy the flake structure of mica and make mica lose its surface luster. Therefore, mica produced by dry process can only be used as filler, and its economic benefit is relatively low. Because water is added in the grinding process of mica powder, the crystal surface luster of mica flakes can be better maintained. Therefore, the utilization of mica powder is wider and the economic value is higher. Superfine mica powder is widely used, among which Pearlescent Mica Powder is the most valuable, which is used as mica for pearlescent pigment. Pearlescent Mica
Powder requires not only fineness, but also natural flake structure and surface finish of mica, so it must be produced by wet process. The traditional wet mill includes roller mill, vibration mill and stirring mill. The crushing way of this kind of mill mostly adopts the crushing way of pressure stress, that is, the crushing of materials occurs under the repeated action of pressure. As a result, the friction on mica is serious, and the flake structure and surface luster of mica will be destroyed to a certain extent. In order to obtain high quality Pearlescent Mica Powder, a new mica wet grinding process is needed. High pressure water jet is a way of breaking tensile stress caused by the expansion of the inherent boundary of particles. That is to say, under the impact of high-speed water jet, the material will produce stress wave propagating to the four directions inside the particles. The stress wave will be reflected at the junction of grains inside the particles, which will cause tensile stress at the junction of grains and cause unloading damage to the material. This characteristic of water jet comminution is especially suitable for the ultra-fine peeling of mica and the preparation of high-quality Pearlescent Mica Powder.

The original ore used in this experiment is from Baotou Baiyun OBO rare earth ore. The original ore of Baiyun OBO was crushed and sampled by chessboard method. Through analysis, the particle size distribution and the content of REO (rare earth oxide) and $\mathrm{Fe}$ in each particle grade of the batch were shown in Table 1.

Table 1. Particle size distribution of rare earth raw ores and distribution of REO and Fe content at each particle size

\begin{tabular}{|c|c|c|c|}
\hline $\begin{array}{c}\text { Grain size } \\
(\mathrm{mm})\end{array}$ & $\begin{array}{c}\text { Yield } \\
(\%)\end{array}$ & $\begin{array}{c}\text { REO } \\
(\mathrm{wt} \%)\end{array}$ & $\begin{array}{c}\text { Fe grade } \\
(\mathrm{wt} \%)\end{array}$ \\
\hline $3 \sim 0.85$ & 18 & 2.51 & 35.22 \\
\hline $0.85 \sim 0.154$ & 44 & 3.56 & 29.32 \\
\hline $0.154 \sim 0.074$ & 16 & 4.27 & 40.21 \\
\hline $0.074 \sim 0.044$ & 7 & 3.98 & 34.58 \\
\hline $0.044 \sim 0.038$ & 3 & 5.52 & 33.45 \\
\hline $0 \sim 0.038$ & 17 & 6.11 & 20.17 \\
\hline
\end{tabular}

\subsection{Experiment process}

The efficiency of the gemstone nozzle used in this experiment is $92 \%$, the working pressure of the water jet is $30,40,50$ and $60 \mathrm{MPa}$ respectively. The velocity of the water jet calculated by the following formula.

$$
V=\sqrt{\frac{2 p}{\rho}}
$$

The results are 226, 260, 291, $319 \mathrm{~m} / \mathrm{s}$. Take 4 raw ores, $1000 \mathrm{~g}$ each, hit them in sequence at different jet speeds. The efficiency of the gemstone nozzle used in this experiment is $92 \%$, and the working pressure of the water jet is 30, 40, 50 and $60 \mathrm{MPa}$ respectively. $\mathrm{V}$ is the outlet water jet velocity $(\mathrm{m} / \mathrm{s}), P$ is the water jet working pressure (PA), and $\rho$ is the water density $\left(\mathrm{kg} / \mathrm{m}^{3}\right)$. The velocity of the effluent jet is $226 \mathrm{~m} / \mathrm{s}, 260 \mathrm{~m} / \mathrm{s}, 291 \mathrm{~m} / \mathrm{s}$ and $319 \mathrm{~m} / \mathrm{s}$ 
respectively. Take 4 parts of raw ore, $1000 \mathrm{~g}$ each, respectively, and strike them in turn at different jet speeds. The crushed mineral particles enter the classification vibrating screen through the discharge port for screening status comparison. The required dissociation of minerals is the percentage of the monomer content of the minerals required in the mineral powder products. The total amount of minerals measured by

$$
F a=\frac{f a}{f a+L a} * 100 \%
$$

$\mathrm{Fa}$ is the monomer dissociation degree of a mineral in the ore crushing and grinding products; $\mathrm{Fa}$ is the monomer content of a mineral in the ore crushing and grinding products; $\mathrm{La}$ is the content of a mineral in its own intergrowth.

\section{Results and discussion}

\subsection{Dissociation effects of different water jet speed}

Under different jet velocity, minerals have different degrees of crushing effect. From the overall trend analysis, it is found that water jet technology has a strong ability of ultra-fine grinding. According to the analysis of mass cumulative percentage diagram, it is found that with the increase of jet velocity, the crushing effect is more obvious. When the particle size is $0 \sim 0.074 \mathrm{~mm}$, the maximum cumulative mineral yield can reach $67.23 \%$, which is $43.23 \%$ higher than that of raw ore. When the jet velocity is $226 \mathrm{~m} / \mathrm{s}$, the cumulative yield of $0-0.074 \mathrm{~mm}$ is $55.12 \%$. With the increase of water flow velocity, the yield of mineral particles in the range of $0 \sim 0.074 \mathrm{~mm}$ increases correspondingly. When the jet velocity is $319 \mathrm{~m} / \mathrm{s}$, the yield of mineral particles in the range of $0 \sim 0.038 \mathrm{~mm}$ is higher than that of other jet velocities. The dissociation degree of rare earth minerals was determined by changing the jet velocity, and the measured monomer and intergrowth were both in section. When the jet velocity is $226 \mathrm{~m} / \mathrm{s}$, the dissociation degree of rare earth minerals is $2.57 \%$. When the jet velocity increases to $319 \mathrm{~m} / \mathrm{s}$, the dissociation degree of rare earth minerals increases to $7.99 \%$, indicating that the water jet comminution technology has a strong dissociation ability for rare earth minerals. With the increase of jet velocity, the content of rare earth minerals $>3 / 4$ increases correspondingly, which is the same as the change trend of monomer dissociation degree. Due to the different jet velocity, the effect of water wedge on the particle's changes, so the particles show different degrees of dissociation and comminution effect.

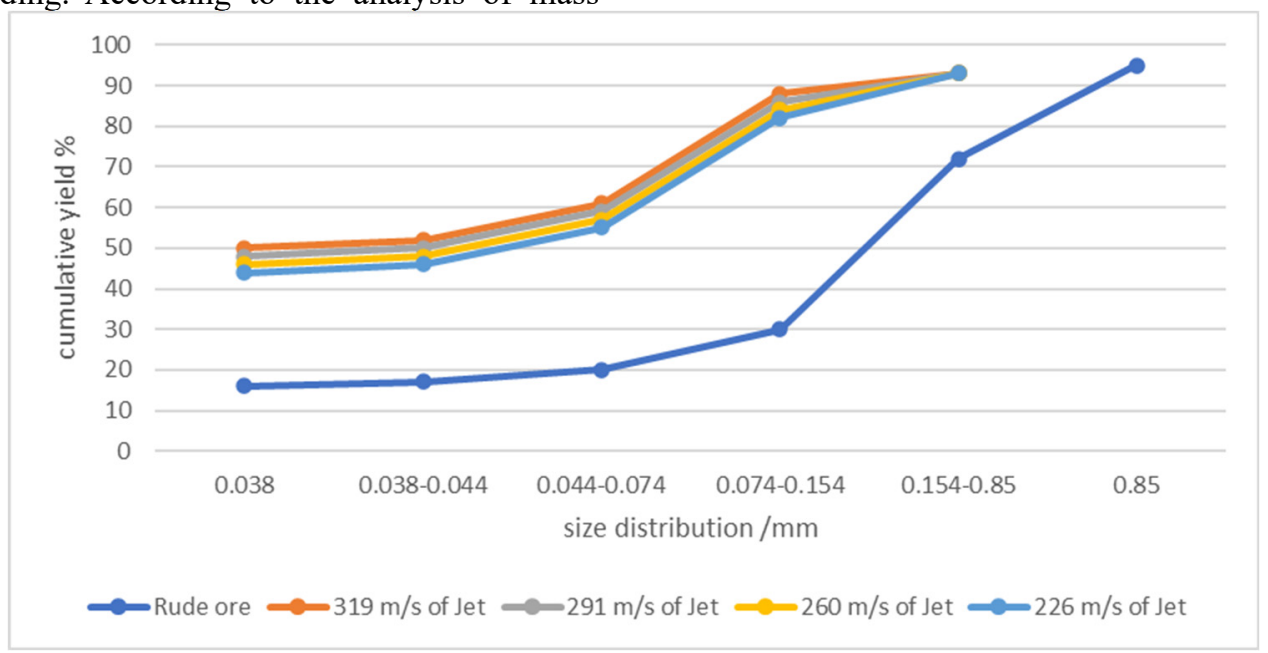

Figure 1. Cumulative mass fraction of crushed product

\subsection{Sample preparation and dissociation degree}

The purpose of the method is to enrich and recover the useful minerals effectively, and separate the useful minerals from the useless minerals. That is, the mineral particles to be recovered are separated by various crushing methods. Generally, after the ore block is broken and ground into powder particle products by mechanical equipment, only one mineral is contained in the mineral particles, or there are many minerals to be recovered and enriched simultaneously. This mineral particle is called monomer. After the massive ore is crushed and ground into powder particles by various crushing equipment, some of them contain only one mineral, which is called the monomer which has been dissociated from the ore; some particles are the associated bodies of minerals, which are coexisted with some useless minerals. In the process of grinding and grinding, the main research content of the analysis of mineral dissociation is the degree of separation of minerals into monomer particles. In mineral powder products, the percentage of the monomer content of the required minerals to the total mineral amount measured in the batch is called the dissociation degree of the required minerals. We mix epoxy resin and curing agent to make solvent in proportion, then add screening to dry the mineral powder, and mix evenly. Because air will be brought in when mixing and adding mineral powder, it is necessary to vacuum and then put it into solidification. Then, after cutting, the prepared ore powder slice shall be set twice according to the above steps, and then treated with coarse grinding, fine grinding, fine grinding and polishing.

The equipment system is in normal operation, the 
connection of each part is reasonable, the overall structure is reasonable, and the system design is basically reasonable, which basically meets the design requirements. The optimal ratio of each equipment in the system includes grinding medium, jet velocity, and feed speed. It needs further verification in the future experiments. The system can be used in a single line and multi-purpose. Other brittle materials can be considered in the future grinding. The crushing rate of materials at all levels is obviously affected by the feed speed, among which the first and third level material crushing rate is the most significant. The grinding medium is too high in quality, and it needs some energy in the process of starting and moving, thus reducing the energy used in grinding and grinding. Through analysis, it is more suitable to adopt Qinhe gold ball and hollow steel ball, which not only reduces the weight of grinding medium, but also ensures the strength and hardness required by design requirements. It is considered to install multi-stage pumps with additional power sources on the spoon box, such as large flow and lift, to provide the energy required for the highspeed operation of grinding medium in the box, or install high speed fan on the side wall of the box to drive the material in the box to move at high speed, so as to achieve the grinding and crushing effect. The device is a small flow jet technology, it is a new processing technology. The characteristics of small flow jet are as follows: small flow rate, narrow slit of jet to material under the same pressure condition. Compared with traditional circular jet, the jet power is low because the required pump volume is small, and the working equipment is easy to move and practical operation is easier. At present, water jet is developing towards fine and fine processing, and the application of small flow jet will be more and more widely.

Table 2. Impact of water jet speed on REO mineral dissociation degree

\subsection{Analyse of dissociation degree}

We use AMICS mineral analysis system to scan each slice surface of each ore sample for 6 frames, and analyse the dissociation of rare earth minerals in ore powder particles. The results show that there are obvious differences in the grade of each fraction of rare earth under different grinding methods, that is, the rare earth concentrate in the fine fraction after water jet grinding, but there is no obvious difference in ball grinding. In order to verify the difference more accurately and intuitively, the ball milled sample whose rare earth grade is closer to the sample distribution after water jet grinding is selected. The dissociation of rare earth minerals in the two samples were detected at the same particle size. The amount of rare earth mineral monomer particles after water jet grinding is obviously higher than that of ball milling. In the case of the same intergrowth of rare earth minerals, after water jet comminution, there are fewer kinds of minerals in the particles, and the dissociation state is better than that of ball milling. Due to the difference of mineral density and hardness, the high-speed jet beam is used to impact the particle instantaneously to produce water wedge tensile effect, which will produce stress concentration at the tip of the mineral particle crack and make the crack increase rapidly, causing the particle to "tear" along the interface of different minerals, and then impact the target at the speed close to the jet beam to achieve the purpose of crushing. Therefore, after water jet comminution, there are more single particles of rare earth minerals. The determination results are shown in Table 2 . The dissociation values of each sample in Table 2 are the weighted average values of Bayan Obo minerals in the screened products.

\begin{tabular}{|c|c|c|c|c|c|c|}
\hline \multirow{2}{*}{ Water jet speed (m/s) } & \multirow{2}{*}{ Number } & Monomer (\%) & \multicolumn{4}{|c|}{ Coenobium (\%) } \\
\cline { 4 - 7 } & & & $4 / 4 \sim 3 / 4$ & $3 / 4 \sim 2 / 4$ & $2 / 4 \sim 1 / 4$ & $<1 / 4$ \\
& & & & & & \\
\hline 226 & 15592 & 2.57 & 25.05 & 10.35 & 31.19 & 30.84 \\
\hline 260 & 16175 & 4.68 & 21.87 & 16.91 & 35.54 & 21.00 \\
\hline 291 & 15821 & 5.70 & 33.53 & 16.17 & 23.04 & 21.56 \\
\hline 319 & 18657 & 7.99 & 37.88 & 18.56 & 16.77 & 18.80 \\
\hline
\end{tabular}

The analysis in Table 2 shows that the monomer dissociation degree of REO mineral increases with the increase of jet velocity. When the jet velocity is $226 \mathrm{~m} / \mathrm{s}$, the dissociation degree of rare earth minerals is $2.57 \%$. When the jet velocity increases to $319 \mathrm{~m} / \mathrm{s}$, the dissociation degree of rare earth minerals increases to $7.99 \%$.

High pressure water jet usually means that liquid water is transformed into high-energy pressure water under the work of high-pressure equipment (high-pressure water pump). Its working pressure is usually up to hundreds of atmospheres, and then it is transformed into high-speed jet beam through a small aperture device. Water jet is a kind of jet which flows out from the nozzle, usually in the form of cylindrical jet beam, and diffuses around through a certain distance. In engineering application, the jet characteristics and working mechanism of high-pressure water jet are very complex, which plays an important guiding role in the research of this paper, but this research only accounts for a part of the work and application of high-pressure water jet machine. In view of the principle and application of the post mixing high-pressure water jet mineral crushing device developed by the research group, this paper mainly focuses on the non-submerged jet in the high-pressure water jet, and analyses the basic characteristics, structure and characteristics of the jet beam. Because of the high-pressure water jet device used in mineral crushing test, it needs to use extremely high pressure. Therefore, a diffusion layer is formed at the edge of the water column at the nozzle outlet. It is found that with the increase of the working pressure of the jet machine, the diffusion degree of the jet beam has different 
increasing trend for the same nozzle.

In the practical application of engineering, there are many factors used to describe the working characteristics of jet machine, including dynamic parameters and structural parameters. According to the dynamic parameters, it can be divided into working pressure, flow rate, power, jet beam velocity and so on. According to the structural parameters, it can be divided into jet head and jet beam width. Considering the characteristics of this research direction, it is decided to convert the working pressure, flow rate and power of the jet machine into the jet beam velocity. For the post mixing high-pressure water jet mineral crushing device, the jet beam velocity usually refers to a high-speed water column formed by highpressure water passing through the nozzle outlet. Due to the strong impact of the high-speed jet beam, the static material particles will produce a stagnation pressure on the material particles. If the stagnation pressure is greater than the compressive strength of the material itself, the material particles will be crushed. However, because mineral materials do not have ductility, their compressive strength is much greater than their tensile strength, commonly known as "compressive but not tensile", so greater stagnation pressure is needed to make the particles compressed and crushed. Combined with the instant of pressure release, the pressure strain energy stored in the mineral particles is released immediately, resulting in the tensile stress failure of the particles. In a word, the compression and pressure release of the material particles will crush the particles. The simultaneous interpreting of the crushing technology of water jet and traditional mechanical crushing means that the most important feature is not only compression and crushing, but also the use of mineral particles themselves, and their tensile strength is far less than that of compressive strength. The high-speed jet beam wedges into the gap space of mineral particles and causes tensile failure of particles.

\section{Conclusions}

Mineral monomer dissociation is the basis and necessary condition of mineral processing. In the grinding process of traditional ball mill, the surface of mineral particles is prone to oxidation reaction, the grinding efficiency is low, and the mineral dissociation effect is not ideal, so it is difficult to obtain high-quality and high-purity ultra-fine powder. The results show that there are differences in water jet comminution. The difference is that the rare earth concentrates in the fine fraction after high pressure water jet comminution, but there is no obvious difference in ball milling. Therefore, water jet crushing is more conducive to the separation and recovery of rare earth minerals.

\section{References}

1. Urakaev F K, Yusupov T S. Numeric Evaluation of Kinematic and Dynamic Characteristics of Mineral Treatment in Disintegrator[J]. Journal of Mining Science, 2017, 53(1): 133-140.

2. Wang J, Jiang T, Liu Y, et al. Influence of microwave treatment on grinding and dissociation characteristics of vanadium titano-magnetite[J]. International Journal of Minerals, Metallurgy, and Materials, 2019, 26(2): 160-167.

3. Sakamoto Y, Nakano Y, Kaneko F, et al. Numerical Simulation of a Laboratory-scale Experiment for the Hydrate Dissociation Process in Porous Media by Acid Injection[J]. International Journal of Offshore and Polar Engineering, 2020, 30(4): 501-512.

4. He H, Cao J, Duan N. Novel bead-milling mechanically pulverized bulk mineral particles to ultrafine scale: Energy storage and cleaner promotion of mineral extraction[J]. Journal of cleaner production, 2018, 198(3): 46-53.

5. Munoz-Santiburcio D, Marx D. Nanoconfinement in slit pores enhances water self-dissociation[J]. Physical review letters, 2017, 119(5): 56-59.

6. Chen X, Espinoza D N. Surface area controls gas hydrate dissociation kinetics in porous media[J]. Fuel, 2018, 234(7): 358-363.

7. Kashim M Z, Tsegab H, Rahmani O, et al. Reaction Mechanism of Wollastonite In Situ Mineral Carbonation for CO2 Sequestration: Effects of Saline Conditions, Temperature, and Pressure $[\mathrm{J}]$. ACS omega, 2020, 5(45): 28942-28954.

8. Jangid A K, Malik P, Singh M. Mineral acid monitored physicochemical studies of oil-in-water nanoemulsions[J]. Journal of Molecular Liquids, 2018, 259(11): 439-452.

9. Tollan P, Hermann J. Arc magmas oxidized by water dissociation and hydrogen incorporation in orthopyroxene[J]. Nature geoscience, 2019, 12(8): 667-671.

10. Li K, Chen J, Peng J, et al. Efficient improvement for dissociation behavior and thermal decomposition of manganese ore by microwave calcination[J]. Journal of Cleaner Production, 2020, 260(1): 121-124.

11. Lazo D E, Dyer L G, Alorro R D. Silicate, phosphate and carbonate mineral dissolution behaviour in the presence of organic acids: A review[J]. Minerals Engineering, 2017, 100(21): 115-123.

12. [12] Cavosie A J, Timms N E, Ferrière L, et al. FRIGN zircon-The only terrestrial mineral diagnostic of high-pressure and high-temperature shock deformation[J]. Geology, 2018, 46(10): 891-894.

13. Laporte S, Pietrucci F, Guyot F, et al. Formic Acid Synthesis in a Water-Mineral System: Major Role of the Interface[J]. The Journal of Physical Chemistry C, 2020, 124(9): 5125-5131.

14. Zhang Y, Liu L, Wang D, et al. Application of lowfield nuclear magnetic resonance (lfnmr) in characterizing the dissociation of gas hydrate in a porous media[J]. Energy \& Fuels, 2021, 35(3): 21742182.

15. Wu Y, Lv Z, Shang L, et al. The stability and coalescence of hydrate in mineral oil emulsion[J]. Petroleum Science and Technology, 2020, 38(18): 883-890.

16. Zhao J, Zhang $\mathrm{G}, \mathrm{Xu} \mathrm{Y}$, et al. Enhancing rate of 
penetration in a tight formation with high-pressure water jet (HPWJ) via a downhole pressurized drilling tool[J]. Journal of Petroleum Science and Engineering, 2019, 174: 1194-1207.

17. Jiang $H$, Zhao $H$, Gao $K$, et al. Numerical investigation of hard rock breakage by high-pressure water jet assisted indenter impact using the coupled SPH/FEM method[J]. Powder Technology, 2020, 376 : 176-186.

18. Ning D, Wang Q, Tian J, et al. Experimental Study on the Coating Removing Characteristics of HighPressure Water Jet by Micro Jet Flow[J]. Micromachines, 2021, 12(2): 173.

19. Fiore N, Caro S, D'Andrea A, et al. Evaluation of bitumen modification with crumb rubber obtained through a high pressure water jet (HPWJ) process[J]. Construction and Building Materials, 2017, 151: 682691.

20. Ayed Y, Germain G. High-pressure water-jet-assisted machining of Ti555-3 titanium alloy: investigation of tool wear mechanisms[J]. The International Journal of Advanced Manufacturing Technology, 2018, 96(1): 845-856.

21. Kang Z, Zhang Z, Deng J, et al. Experimental Research of High-Temperature and High-Pressure Water Jet Characteristics in ICRC Engine Relevant Conditions[J]. Energies, 2019, 12(9): 1763.

22. Zhou M, Liu H, Kang C, et al. Resistance of curved surfaces to the cavitation erosion produced through high-pressure submerged waterjet[J]. Wear, 2019, 440: 203091.

23. Latchoumi T P, Balamurugan K, Dinesh K, et al. Particle swarm optimization approach for waterjet cavitation peening[J]. Measurement, 2019, 141: 184189.

24. Perec A. Experimental research into alternative abrasive material for the abrasive water-jet cutting of titanium[J]. The International Journal of Advanced Manufacturing Technology, 2018, 97(1): 1529-1540.

25. Chen X, Li X, Song W, et al. Effects of a low-pressure water jet assisting the laser etching of polycrystalline silicon[J]. Applied Physics A, 2018, 124(8): 1-14. 